\title{
The Study on Compound Drought and Heatwave Events in China Using Complex Networks
}

\author{
Kaiwen $\mathrm{Li}^{1,2} \mathbb{D}$, Ming Wang ${ }^{1,3, *}$ and Kai Liu ${ }^{1,3} \mathbb{D}$ \\ 1 Academy of Disaster Reduction and Emergency Management, Beijing Normal University, \\ Beijing 100875, China; 202031051092@mail.bnu.edu.cn (K.L.); liukai@bnu.edu.cn (K.L.) \\ 2 Faculty of Geographical Science, Beijing Normal University, Beijing 100875, China \\ 3 The School of National Safety and Emergency Management, Beijing Normal University, Beijing 100875, China \\ * Correspondence: wangming@bnu.edu.cn; Tel./Fax: + 86-10-5880-6497
}

check for updates

Citation: Li, K.; Wang, M.; Liu, K. The Study on Compound Drought and Heatwave Events in China Using Complex Networks. Sustainability 2021, 13, 12774. https://doi.org/ $10.3390 /$ su132212774

Academic Editors: Xiaodong Yan, Jia Yang and Shaofei Jin

Received: 28 October 2021

Accepted: 16 November 2021

Published: 18 November 2021

Publisher's Note: MDPI stays neutral with regard to jurisdictional claims in published maps and institutional affiliations.

Copyright: (c) 2021 by the authors. Licensee MDPI, Basel, Switzerland. This article is an open access article distributed under the terms and conditions of the Creative Commons Attribution (CC BY) license (https:/ / creativecommons.org/licenses/by/ $4.0 /)$.

\begin{abstract}
Compound extreme events can severely impact water security, food security, and social and economic development. Compared with single-hazard events, compound extreme events cause greater losses. Therefore, understanding the spatial and temporal variations in compound extreme events is important to prevent the risks they cause. Only a few studies have analyzed the spatial and temporal relations of compound extreme events from the perspective of a complex network. In this study, we define compound drought and heatwave events (CDHEs) using the monthly scale standard precipitation index (SPI), and the definition of a heatwave is based on daily maximum temperature. We evaluate the spatial and temporal variations in CDHEs in China from 1961 to 2018 and discuss the impact of maximum temperature and precipitation changes on the annual frequency and annual magnitude trends of CDHEs. Furthermore, a synchronization strength network is established using the event synchronization method, and the proposed synchronization strength index (SSI) is used to divide the network into eight communities to identify the propagation extent of CDHEs, where each community represents a region with high synchronization strength. Finally, we explore the impact of summer Atlantic multidecadal oscillation (AMO) and Pacific decadal oscillation (PDO) on CDHEs in different communities. The results show that, at a national scale, the mean frequency of CDHEs takes on a non-significant decreasing trend, and the mean magnitude of CDHEs takes on a non-significant increasing trend. The significant trends in the annual frequency and annual magnitude of CDHEs are attributed to maximum temperature and precipitation changes. AMO positively modulates the mean frequency and mean magnitude of CDHEs within community 1 and 2, and negatively modulates the mean magnitude of CDHEs within community 3. PDO negatively modulates the mean frequency and mean magnitude of CDHEs within community 4. AMO and PDO jointly modulate the mean magnitude of CDHEs within community 6 and 8. Overall, this study provides a new understanding of CDHEs to mitigate their severe effects.
\end{abstract}

Keywords: compound drought and heatwave events; complex network; event synchronization; atmospheric circulation patterns

\section{Introduction}

Global warming has led to an increase in drought and heatwave events [1,2]. The conjoined extreme events of droughts and heatwaves are considered as compound drought and heatwave events (CDHEs) [3-5], which have severe impacts on socioeconomic development and the environment. For example, severe drought and heatwaves in the 2003 European summer resulted in around 40,000 deaths [6]. Drought and heat anomalies in the central United States has caused at least USD 30 billion in damages [7]. Therefore, it is essential to study the spatial and temporal variations in, and spatial influences of, CDHEs to mitigate their effects.

There is no unified standard for the definition of CDHEs, because drought events and heatwave events correspond to a variety of definitions. Drought events are usually defined 
by various indicators, such as the standardized precipitation index (SPI) $[8,9]$, the standardized precipitation evapotranspiration index $[10,11]$, and the Palmer drought severity index $[12,13]$. Heatwave events are mainly defined by relative thresholds (percentile thresholds), absolute thresholds, and durations [14,15]. In recent studies, the definition of CDHEs mainly refers to the combination of drought and heatwave event definitions mentioned above [4,16-18]. There are also studies that directly describe the properties of CDHEs by corresponding metrics. For instance, Wu et al. [19] proposed a dry-hot magnitude index (DHMI) to characterize the magnitude of CDHEs. Their results show that highmagnitude CDHEs mainly occur in northeastern and southwestern China. Wu et al. [20] derived a standardized compound event indicator and a standardized dry-hot index to evaluate the severity of changes in CDHEs in the warm season. Their results show that the severity of CDHEs increases significantly in most parts of China, and temperature is the dominant driving factor compared with precipitation. The variation in various properties (frequency, magnitude, severity, etc.) of CDHEs, and the potential linkage of such variation to atmospheric circulation patterns, has also been investigated. Hao et al. [16] applied a logistic regression model to examine the relation between the occurrence of compound events and El Niño-Southern Oscillation (ENSO) represented by NINO34. The results show that ENSO plays an important role in the occurrence of CDHEs during the warm season in the northern parts of South America, southern Africa, southeastern Asia, and Australia. In China, there has been a significant increase in the frequency and spatial extent of compound dry-warm events, which is partly related to atmospheric circulation patterns [21]. Mukherjee et al. [22] used a Poisson generalized linear model to analyze the relation between the frequency of seasonal CDHEs and the warm and cold phases of ENSO, Pacific Decadal Oscillation (PDO), and North Atlantic Oscillation (NAO). The results show that ENSO is strongly related with CDHEs over the southern hemisphere, PDO influences the frequency of CDHEs over western North America during the boreal summer, while NAO is weakly related with CDHEs. The composite analysis, correlation analysis, and logistic regression model have all been used to investigate the influence of various atmospheric circulation patterns on CDHEs in China. These methods found that the Atlantic Multidecadal Oscillation (AMO) affects CDHEs for about $18.52 \%$ of mainland China, while NAO and PDO, respectively, account for around $14.64 \%$ and $12.96 \%$, and ENSO affects CDHEs by about $5.27 \%$ [23]. In general, previous studies analyzed the spatial and temporal variations in properties of CDHEs using various definitions or metrics, revealing that atmospheric circulation patterns have a profound impact on CDHEs.

The complex systems theory applied to the study of extreme events has received increased attention. Boers et al. introduced network divergence to directed networks to predict extreme rainfall events in the central Andes [24]. Complex networks have been used to reveal global patterns of extreme rainfall teleconnections, and Rossby waves were shown to be the physical mechanism of these teleconnections [25]. Konapala and Mishra [26] applied the complex network approach to study the spatial and temporal evolution of droughts in the continental USA. They found that drought events propagate differently at different thresholds associated with their initiation. However, complex network approaches have rarely been applied to the study of CDHEs.

The objective of this study is to evaluate the spatiotemporal variations and propagation extent of CDHEs for the period of 1961-2018 in China, based on monthly SPI, daily maximum temperature, and a complex network approach. The correlations between AMO, $\mathrm{PDO}$, and the frequency and magnitude of CDHEs are employed to explore the possible physical mechanism of the formation of CDHEs. The results of this study will enhance our understanding of compound extreme events and provide suggestions for decisionmakers to mitigate the negative impacts of these events in China. 


\section{Data and Definition}

\subsection{Data}

Our study is based on monthly precipitation data and daily maximum temperature data from the CN05.1 dataset [27]. The time span of precipitation and maximum temperature data is from 1961 to 2018 , the resolution is $0.25^{\circ} \times 0.25^{\circ}$, and there are 163 grid points in the north-south direction and 283 grid points in the east-west direction, where the number of grid points within China is 15,247 (precipitation and maximum temperature data for Taiwan Province are missing).

AMO and PDO are major large-scale circulation patterns that influence the climate of East Asia. The AMO index is defined as the average anomalies of sea surface temperature (SST) in the north Atlantic [28]. Its positive (negative) phase usually indicates that SST is warmer (cooler) than the average SST across the north Atlantic Ocean. The PDO index is defined as the leading principal component of the north Pacific (NP) monthly SST anomalies [29]. Its positive (negative) phase corresponds to cool (warm) SST in the central and western NP. In this study, we found that more than $83.5 \%$ of CDHEs occur during June, July, and August (JJA) in China (as shown in Figure 1); thus, we focused on the impact of the AMO and PDO indexes in JJA on CDHEs, where the temporal series of the AMO and PDO indexes in JJA are derived from the China National Climate Center (http: / / cmdp.ncc-cma.net, accessed on 18 November 2021), and shown in Figure 2.

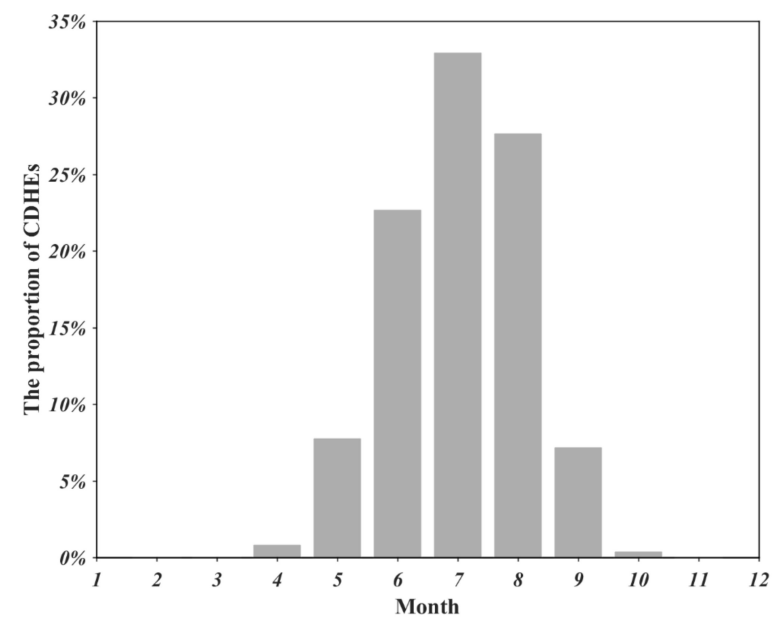

Figure 1. The proportion of CDHEs in different months for the period of 1961-2018.

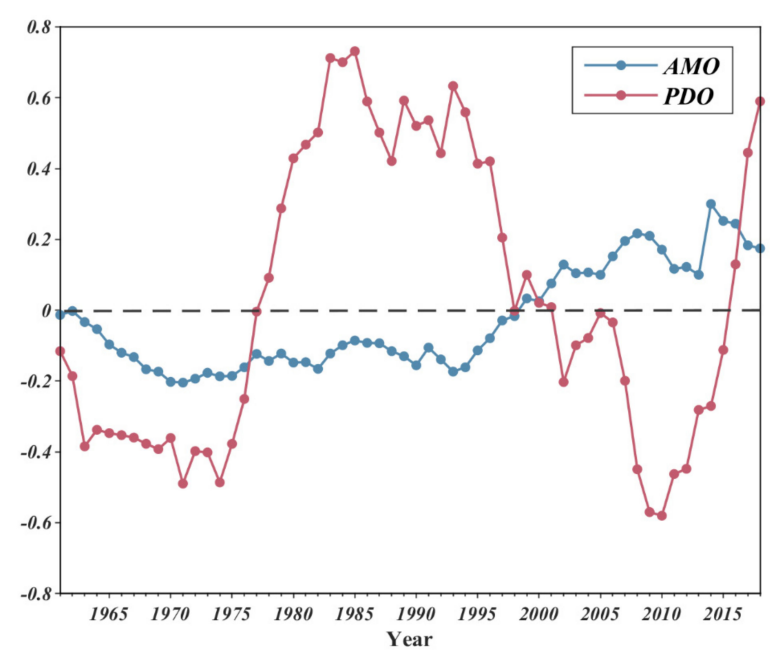

Figure 2. Temporal series of AMO (blue) and PDO (red) index during JJA for the period of 1961-2018 after 9 year moving average. 


\subsection{Defining Compound Drought and Heatwave Events}

CDHEs are defined as extreme disaster events of drought in conjunction with a heatwave. The influences of drought events are usually long-lasting, so the monthly SPI is calculated to characterize the degree of drought in a given month [11], and -1 is selected as the threshold of drought conditions. According to the World Meteorological Organization's recommended criteria for heatwaves [30], we define a heatwave as an event with a daily maximum temperature $\left(\mathrm{T}_{\max }\right) \geq 32{ }^{\circ} \mathrm{C}$ and a duration of $\geq 3$ consecutive days. As shown in Figure 3, the occurrence of a CDHE can be defined as a binary variable, where 1 is for occurrences and 0 is for non-occurrences. $\Delta t$ represents the duration of a CDHE, and $\sum \Delta T$ represents the accumulative temperatures of a CDHE, which are the sum of the daily maximum temperature exceeding the threshold for the duration of a CDHE. The SPI of the month in which the CDHE is located, and its accumulative temperatures are used for the subsequent calculation of DHMI. More details are described in Section 3.1.

Compared with previous studies, we focused not only on the interannual variation in the frequency of CDHEs, but also on the interannual variation in the magnitude of CDHEs, where the magnitude of CDHEs is represented by DHMI.

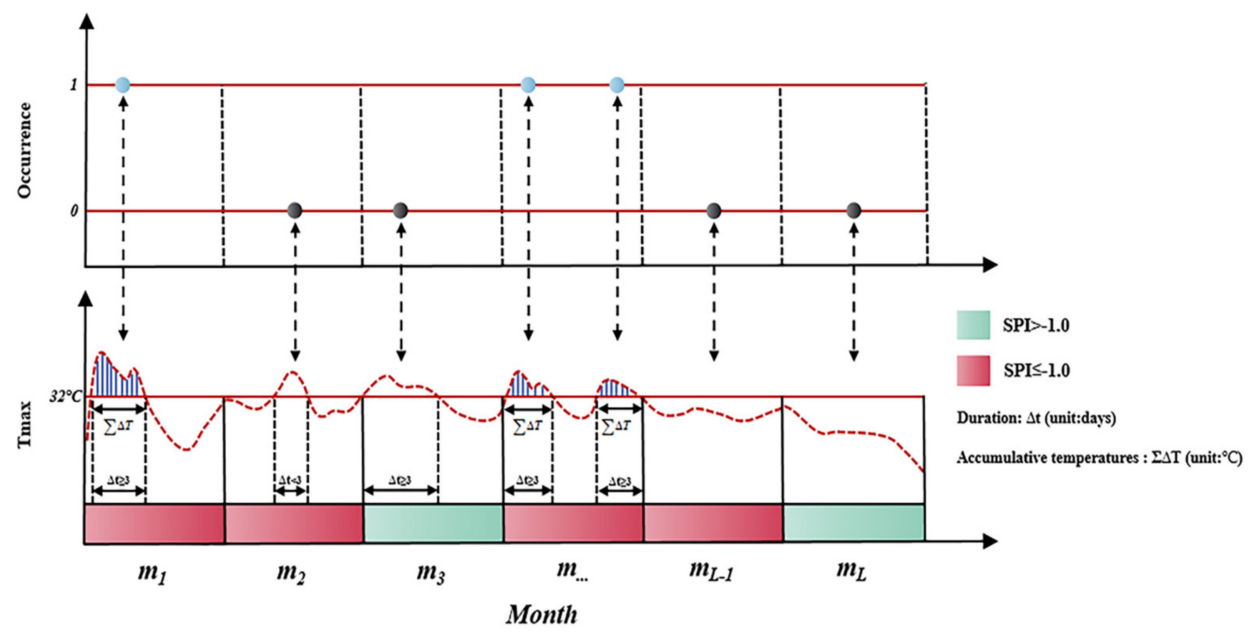

Figure 3. The schematic diagram of CDHEs. L is the total number of months for the period of 1961-2018. The top y-axis represents the occurrence of CDHEs, 1 for occurrences and 0 for nonoccurrences. The bottom y-axis represents the maximum temperature. $\Delta t$ represents the duration of a CDHE and $\sum \Delta T$ represents the accumulative temperatures of a CDHE.

\section{Methodology}

\subsection{The Drought-Heatwave Magnitude Index}

The magnitude of a CDHE is jointly determined by drought degree and heatwave conditions; then, the DHMI of a CDHE can be defined as [19]:

$$
\mathrm{DHMI}=\mathrm{P}\left(\sum \Delta T\right) \times(\Delta S P I)
$$

where $\sum \Delta T$ represents the accumulative temperatures of a CDHE, $\mathrm{P}\left(\sum \Delta T\right)$ is obtained using a nonparametric method to estimate the cumulative density function (CDF) of $\sum \Delta T$, transforming $\sum \Delta T$ to a non-exceedance probability ranging from 0 to 1 . A larger $\sum \Delta T$ value corresponds to a higher probability of $\mathrm{P}\left(\sum \Delta T\right)$ [31]. The degree of drought is represented by monthly SPI. $\triangle$ SPI proposed by the runs theory $[32,33]$ is the absolute value of the difference between the SPI for the month in which the CDHE occurs and the drought threshold. Therefore, a larger $\triangle S P I$ indicates a more severe drought. DHMI can also be treated as a weighted value of $\triangle S P I$, so its maximum value is the maximum value of $\triangle S P I$. 


\subsection{Event Synchronization}

The method of event synchronization is used to reveal the global pattern of extreme rainfall teleconnections [25] and predict extreme floods [24]. In this study, grid $i$ and grid $j$ are selected to describe the definition of synchronized events. We suppose that, for grid $i$, a CDHE occurs at a moment $t_{m}^{i}$; for grid $j$, a CDHE occurs at a moment $t_{n}^{j}$, where $m \in[1, \mathrm{M}]$, and $n \in[1, \mathrm{~N}] . \mathrm{M}$ and $\mathrm{N}$ denote the total number of CDHEs at grids $i$ and $j$, respectively [34]. CDHEs occurring on consecutive days are counted as single events, and the occurrence time of each CDHE is determined as the time corresponding to the first day when the event occurs. A dynamical delay $\tau_{m, n}^{i, j}$ is introduced to decide whether a pair of events occurs at $t_{m}^{i}$, and $t_{n}^{j}$ is counted as a synchronized event. Its definition is as follows:

$$
\tau_{m, n}^{i, j}=\min \left(\frac{\left\{t_{m+1}^{i}-t_{m}^{i}, t_{m}^{i}-t_{m-1}^{i}, t_{n+1}^{j}-t_{n}^{j}, t_{n}^{j}-t_{n-1}^{j}\right\}}{2}\right)
$$

Furthermore, we introduce a maximum delay ( $\tau_{\max }=2$ days) to constrain the formation of CDHEs [24]. $f(i / j)$ is used to define the number of times a CDHE shortly occurs in grid $i$ after it occurs in grid $j$, i.e:

$$
f(i / j)=\sum_{m=1}^{M} \sum_{n=1}^{N} S_{i j}
$$

with

$$
S_{i j}=\left\{\begin{array}{l}
1 \quad \text { if } 0<t_{m}^{i}-t_{n}^{j} \leq \min \left(\tau_{m, n}^{i, j}, \tau_{\text {max }}\right) \\
1 / 2 \text { if } t_{m}^{i}=t_{n}^{j} \\
0 \quad \text { else }
\end{array}\right.
$$

and analogously for $f(j / i) . Q_{i j}$ denotes the synchronization strength between grids $i$ and $j$. Its definition is as follows

$$
Q_{i j}=\frac{f(i / j)+f(j / i)}{\sqrt{M \times N}}
$$

where $Q_{i j}$ is normalized to $Q_{i j} \in[0,1]$. There is $Q_{i j}=1$ if CDHEs are fully synchronized between grids $i$ and $j$.

\subsection{Network Construction}

In this study, the synchronization strength network is established through traversing the synchronization strength for all possible pairs of grid points, where the grid points are defined as nodes, and the synchronization strength between grid points is the weight of the connected edges in the network. To eliminate minor edges, we prune the network using $Q_{95}$, corresponding to the 95th percentile of all synchronization strengths. Take grids $i$ and $j$ as examples: there is a connected edge between grids $i$ and $j$ if $Q_{i j} \geq Q_{95}$. Finally, we extract some edges with higher weights through the abovementioned principle. The total number of edges in the synchronization strength network is 2.496 million, which also indicates that the network has a large scale.

\subsection{Complex Network Metrics}

The establishment of the synchronization strength network provides a possibility to identify the propagation extent of CDHEs. Complex network metrics aim to quantitatively describe the topological characteristics of nodes and edges in the network.

The degree centrality is a measurement to evaluate the local importance of nodes in the network, and the degree value of node $i$ is defined as:

$$
k_{i}=\sum_{j=1}^{\theta} A_{i j}
$$


with

$$
A_{i j}=\left\{\begin{array}{c}
1, \text { if node } i \text { and } j \text { is connected } \\
0, \text { otherwise }
\end{array}\right.
$$

where $k_{i}$ is the degree value of node $i, \theta$ is the total number of nodes, $A_{i j}$ is the adjacency matrix, and $A_{i j}=1$ if node $i$ and node $j$ are connected; otherwise, $A_{i j}=0$. A higher degree value for a node indicates that the node is connected to more nodes that are strongly synchronized with its CDHEs.

The average distance of nodes is introduced to describe the average status of the geographic distance of all edges for a node, and its mathematical expression is given as:

$$
A D_{i}=\frac{\sum\left(D_{i j} \times A_{i j}\right)}{k_{i}}
$$

where $A D_{i}$ is the average distance of node $i$ and $D_{i j}$ is the geographic distance between node $i$ and node $j$. The average distance of nodes is concerned with whether a single node has the possibility of connecting to distant nodes.

For node $i$, the synchronization strength index $S S I_{i}$ is proposed to quantify the ability of node $i$ to form a region with high synchronization strength. In general, the regions with high synchronization strength that are formed should satisfy three conditions: the degree of nodes in the region is larger, the average distance between these nodes and its neighboring nodes is shorter, and the synchronization strength between neighboring nodes is stronger. Thus, the definition of $S S I_{i}$ is given as:

$$
\operatorname{SSI}_{i}=\text { normalized }\left(\frac{k_{i} \times C C_{i}}{A D_{i}}\right)
$$

with

$$
C C_{i}=\frac{2 \times E_{\text {existed }}}{k_{i} \times\left(k_{i}-1\right)}
$$

where $C C_{i}$ is the clustering coefficient of node $i, E_{\text {existed }}$ is the number of existing edges between neighboring nodes of node $i$, and $C C_{i}$ is used to measure the clustering degree of node $i$ in the network $[35,36]$. A node with a larger SSI is more likely to form an initial region with high synchronization strength, and the occurrence of CDHEs in the region is more synchronous.

\subsection{Community Detection}

Community detection is a method for discovering closely related components in a network, and it is utilized to identify the propagation extent of CDHEs. The synchronization strength between the nodes within the community is stronger; conversely, the synchronization strength is weaker between different communities. The existing algorithms of community detection mainly include the fast Newman and GN algorithms for unweighted networks [37,38], and the spin glass algorithm, random walk algorithm, and label propagation algorithm for weighted networks [39,40]. The spin glass algorithm, developed from the theory of spin glass in physics, is suitable for weighted networks with a larger scale, and is used to divide communities in the synchronization strength network. The mechanism of this algorithm can be briefly described as the connection or disconnection between nodes, and the community structure of the network is interpreted as the spin configuration that minimizes the energy of spin glass [41]. The communities derived from the spin glass algorithm are cohesive subgraphs.

For community detection, the modularity Mod is introduced to compare the division quality under different numbers of communities. The definition of modularity Mod is given as [42]:

$$
\operatorname{Mod}=\frac{1}{2 e_{w}} \sum_{i, j}\left[w_{i j}-\frac{w_{i} * w_{j}}{2 e_{w}}\right] \delta\left(C_{i}, C_{j}\right)
$$


where Mod is the global weighted modularity, $\operatorname{Mod} \in[0,1] . e_{w}$ is the sum of the weights of all edges, $w_{i j}$ is the weight of the edge between node $i$ and node $j, w_{i}$ is the sum of the weights of edges connected to node $i$, and analogously for $w_{j}$. A higher Mod indicates that the nodes within each community are more closely related and more divergent between the nodes of different communities. $C_{i}$ and $C_{j}$ are the communities of node $i$ and node $j$, respectively. If node $i$ and node $j$ belong to the same community, $C_{i}=C_{j}, \delta\left(C_{i}, C_{j}\right)=1$; otherwise, $\delta\left(C_{i}, C_{j}\right)=0$.

\section{Results and Discussions}

The annual frequency and annual magnitude of CDHEs are examined in this study. The annual frequency of CDHEs is defined as the total number of heatwaves in drought months of each year, while the annual magnitude of CDHEs is calculated as the mean DHMI of all CDHEs occurring in the same year. In Section 4.1, we first analyze the linear trend in the mean frequency and mean magnitude of CDHEs over all grids during 1961-2018. For spatial variation, we calculate the mean annual frequency and mean annual magnitude of CDHEs, and then analyze the trends in the annual frequency and annual magnitude of CDHEs in each grid based on the linear fitting method for the period of 1961-2018. In Section 4.2, the synchronization strength index (SSI) is proposed to initially identify the number of communities and the spin glass algorithm is used to derive the propagation extent of CDHEs. We also explore the influence of AMO and PDO on the variations in the frequency and magnitude of CDHEs in each community.

\subsection{Spatiotemporal Variation Analysis of CDHEs}

From a national perspective, as shown in Figure 4, the trend in the mean frequency of CDHEs over all grids is $-9.645 \times 10^{-4} \mathrm{yr}^{-1}$ with $p=0.401$ (Figure $4 \mathrm{a}$ ), and the trend in the mean magnitude of CDHEs over all grids is $7.991 \times 10^{-6} \mathrm{yr}^{-1}$ with $p=0.959$ (Figure $4 \mathrm{~b}$ ); these values indicate a non-significant $(p>0.05)$ decrease in the mean frequency of CDHEs, as well as a non-significant increase in the mean magnitude of CDHEs.

We further investigated the spatial distribution of the characteristics of CDHEs between different regions, the mean annual frequency and mean annual magnitude of CDHEs in each grid are presented in Figure 5a,b; we found that the grids where CDHEs had not occurred from 1961 to 2018 were mainly located at high altitudes of the Chinese mainland (as shown in Figure 5c). Because high-altitude areas are relatively cold, air temperature struggles to surpass the threshold temperature corresponding to the definition of heatwaves, which is not conducive to the formation of CDHEs. The spatial distribution of the mean annual frequency of CDHEs is shown in Figure 5a. It can be seen that the mean annual frequency of CDHEs in Northeast China (NEC) and the middle-eastern region of North China (NC) is less than 1, while the mean annual frequency in the western region of Northwest China (NWC), the western region of NC, the eastern region of Southwest China (SWC), most regions of central China (CC), East China (EC), and South China (SC) is more than 1; the mean annual frequency of CDHEs in southern Guangxi province and northern Hainan province exceeds 2 , and the maximum mean annual frequency is 2.6. As shown in Figure $5 b$, we found that the spatial distribution pattern of the mean annual magnitude of CDHEs in the northern China (including NC, NEC, and NWC) is relatively similar to that of the mean annual frequency, and the mean annual magnitude of CDHEs reaches a maximum (0.27) in the eastern region of Xinjiang province. The mean annual magnitude exceeds 0.2 in the western Chongqing province, southern Guangxi and Jiangxi provinces, and northern Hainan province. 

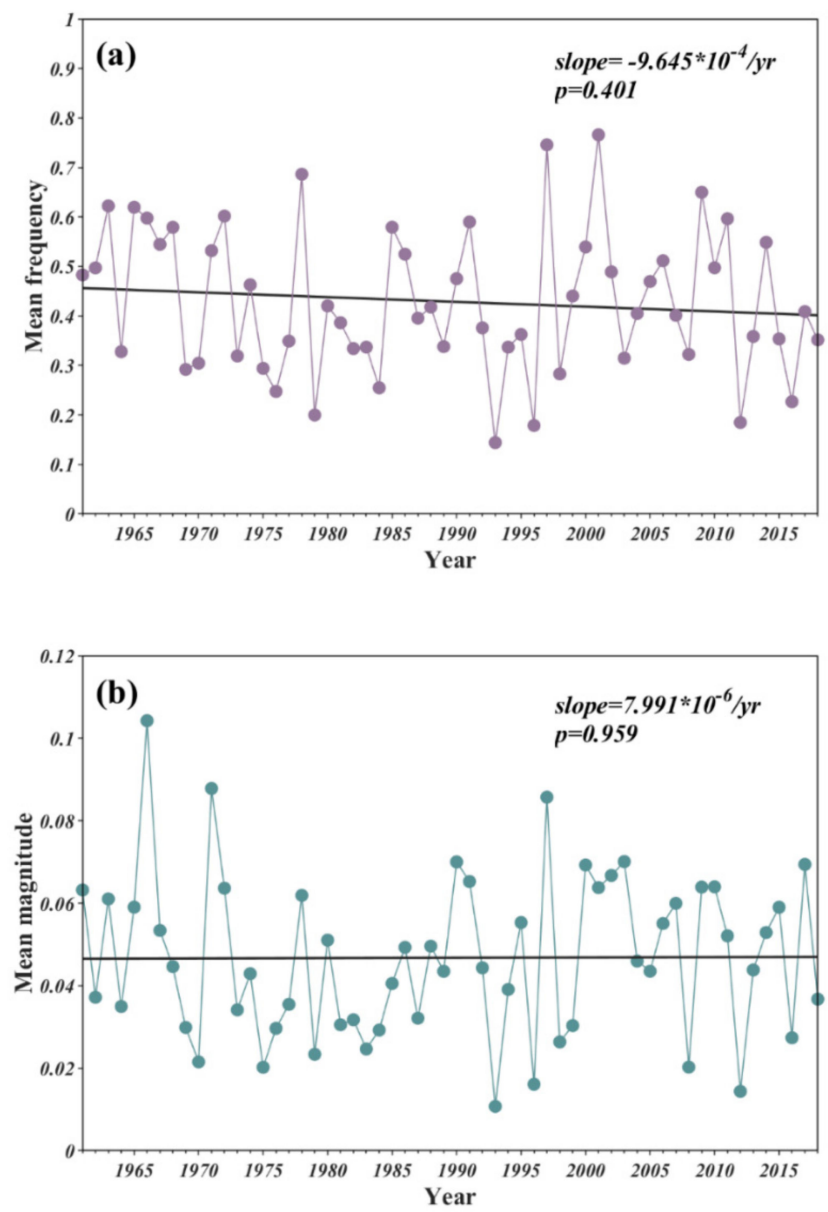

Figure 4. Temporal series of the mean frequency (a) and mean magnitude (b) of CDHEs over all grids for the period of 1961-2018 in China. Black solid lines represent the results of linear fitting.

The joint analysis of the mean annual frequency and mean annual magnitude of CDHEs shows that the high mean annual frequency and high mean annual magnitude of CDHEs in the western region of NWC and NC may be attributed to the fact that the corresponding grid points are located in the desert, where the continuous high temperature and precipitation deficit are more conducive to the formation of CDHEs, which also makes the magnitude of CDHEs higher. The reason for the low mean annual frequency and mean annual magnitude in NEC and the middle-eastern region of NC is the low maximum temperature in the region $[43,44]$, which inhibits the formation of CDHEs and also makes the accumulative temperatures of CDHEs lower than those of CDHEs in other regions. Except for the northern China, the spatial distribution patterns of mean annual frequency and mean annual magnitude of CDHEs are dissimilar. At low latitudes, the number of drought months probably determines the mean annual frequency of CDHEs due to high maximum temperatures. The accumulative temperatures of CDHEs and the drought degree of the month in which CDHEs occur together determine the mean annual magnitude of CDHEs.

Trends in the annual frequency and annual magnitude of CDHEs in each grid are shown in Figure 6a,b. There is a significant increase in the annual frequency of CDHEs in NC, as well as in some parts of NWC. This may be due to the effect of unchanged precipitation and increased maximum temperature during JJA in these regions, as shown in Figure $6 c, d$. The significant increase in maximum temperature is more likely to lead to heatwaves. Therefore, the significant increase in the annual frequency of CDHEs in these regions can be attributed to the rise in maximum temperature. The regions where the annual frequency of CDHEs decreases significantly are mainly located in the western region of NWC, the northern region of $\mathrm{CC}$, the western region of $\mathrm{EC}$, and the southern 
region of SC. In the western region of NWC, which is located in the desert and has an environment characterized by a high temperature, there is potential for more heatwaves. A significant increase in precipitation in this region makes it possible to convert from drought to non-drought months and may lead to a significant decrease in the annual frequency of CDHEs in this region. The possible mechanism for this in the southern region of SC is similar to that of the western region of NWC. The reason for the significant decrease in the annual frequency of CDHEs in the southern region of $\mathrm{SC}$ is a significant increase in precipitation. In the northern region of $\mathrm{CC}$ and the western region of $\mathrm{EC}$, unchanged precipitation and a decreased trend in maximum temperature are generally consistent with the results of previous studies [20,45], and likely inhibit the formation of CDHEs in these regions.
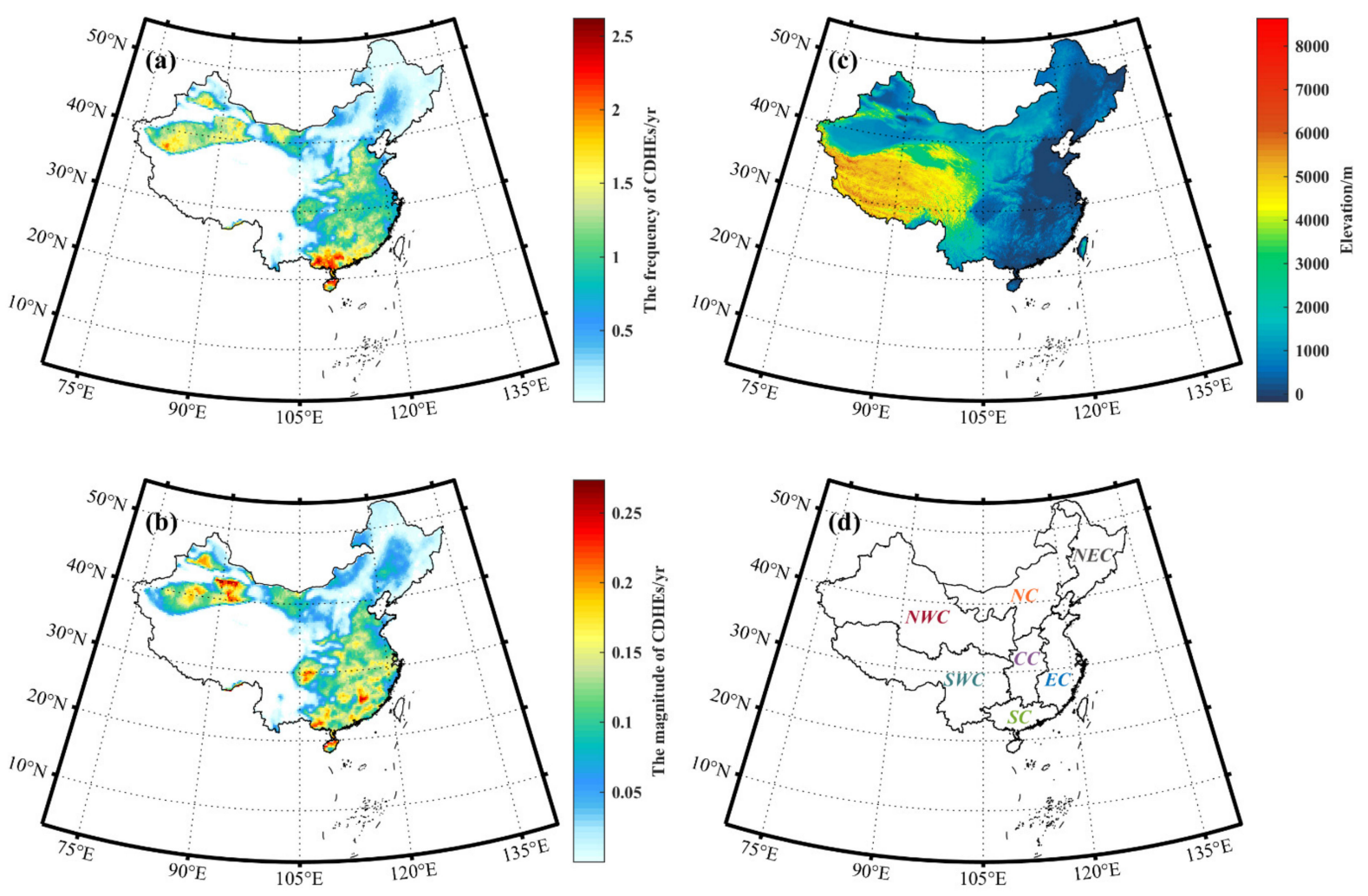

Figure 5. The spatial distribution of the mean annual frequency (a), mean annual magnitude (b) of CDHEs for the period of 1961-2018, elevation (c) and seven regions (d) in China.

According to the definition of the annual magnitude of CDHEs, the annual magnitude trend is likely affected by the individual or combined effect of variations in precipitation and temperature. For instance, with precipitation unchanged, the annual magnitude of CDHEs is expected to increase with the increase in maximum temperature. In some parts of NWC and NC, the annual magnitude shows a significantly increased trend. The precipitation decreases significantly, and the maximum temperature shows a significant increase in the eastern region of $\mathrm{NC}$, which may cause an increase in $\triangle S P I$ and accumulative temperatures, resulting in a significant increase in the annual magnitude of CDHEs in this region. Except for the eastern region of NC, the interannual precipitation is unchanged, and the significantly increased maximum temperature dominates the significant increase in the annual magnitude of CDHEs. The regions showing a significant decrease in the annual magnitude of CDHEs are mainly located in the western region of NWC, CC, and the eastern region of SWC, where the western region of NWC and the eastern region of SWC show a significant increase in precipitation and a non-significant increase in maximum temperature; as a result, a significant increase in precipitation probably becomes the main factor that leads to a significant decrease in the annual magnitude. Precipitation in the northern and 
central regions of CC shows a non-significant increase, but the maximum temperature in the former shows a non-significant decrease; the combined effect of maximum temperature and precipitation may contribute to the significant decrease in the annual magnitude of CDHEs, while the maximum temperature in the latter shows a non-significant increase; thus, the main reason for the significant decrease in the annual magnitude of CDHEs is that the change in the annual magnitude in this region is more sensitive to changes in precipitation than it is to maximum temperature.
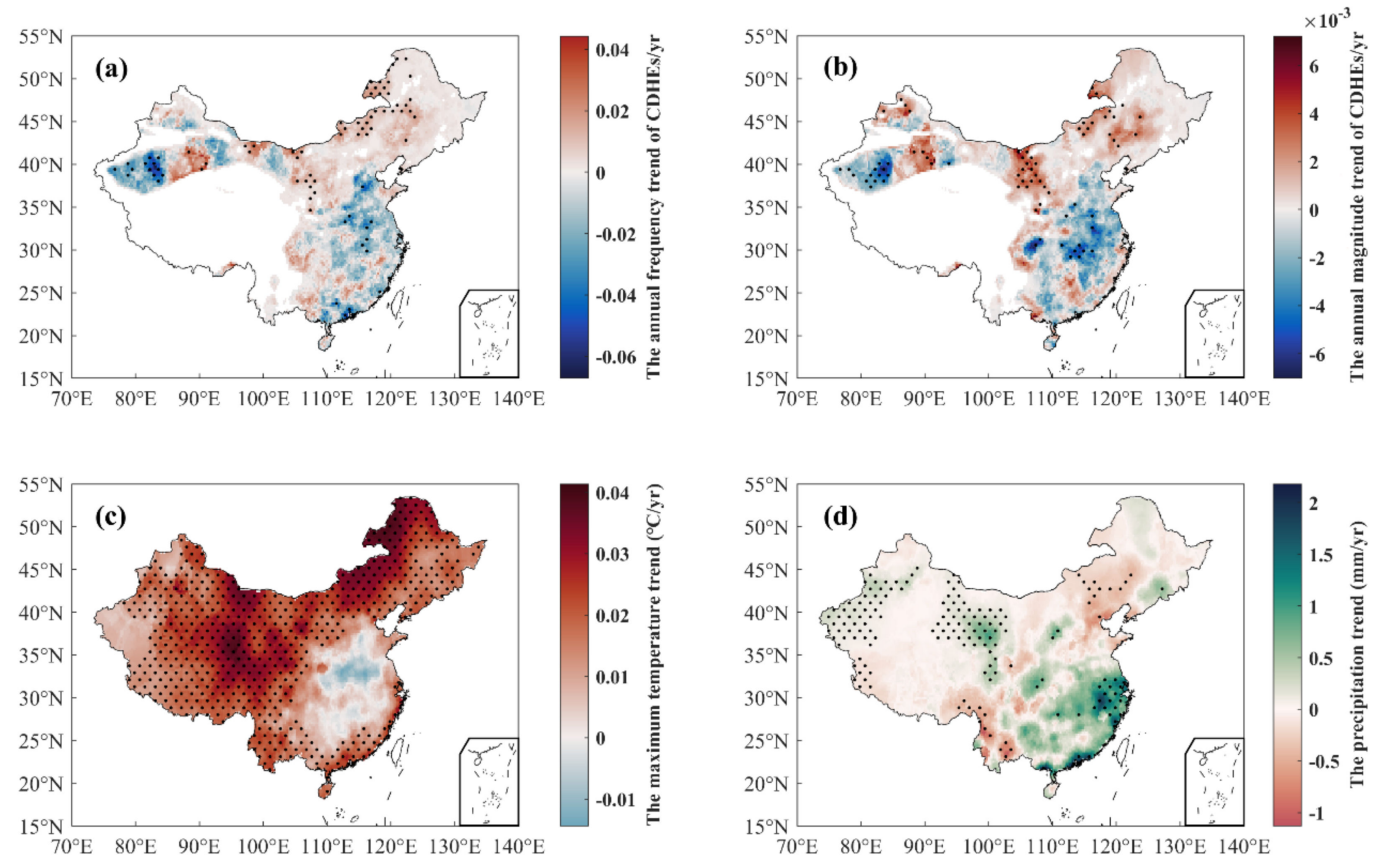

Figure 6. Trend analysis of the annual frequency (a) and annual magnitude (b) of CDHEs, and the maximum temperature (c) and precipitation (d) during JJA for the period of 1961-2018 in China. Black dots indicate statistical significance at a 0.05 significance level.

\subsection{The Propagation Extent and Driving Factors of CDHEs}

In this study, we use the method of event synchronization to establish the synchronization strength network, which is applied to reveal the synchronism of the occurrence time of CDHEs among nodes. A higher synchronization strength between a pair of nodes indicates that there are more CDHEs occurring at similar time points, and it also implies that the total number of CDHEs between nodes is similar. If a region exists in which the relations among nodes are relatively close, the occurrence time of CDHEs in the past may be similar in this region, and CDHEs are more likely to propagate within the region. In order to find the abovementioned regions, we propose an SSI based on the degree value, as well as the average distance and clustering coefficient of nodes in complex networks. The SSI of nodes is shown in Figure 7.

We found six regions that are formed by some nodes with high $S S I(S S I \in[0.6,1.0])$. According to the definition of SSI, these six regions are initially identified as core regions where CDHEs can easily propagate. However, this method does not provide a clear boundary of the propagation extent of CDHEs. The spin glass algorithm, a nonoverlapping algorithm of community detection, is used to detect closely related components in the network. In comparison with the connected edges between communities, the connected edges between nodes within a community are denser and have higher weights; thus, each community can be considered as the propagation extent of CDHEs. In this study, the number of communities is initially determined to be four to eight, with reference to the number of core regions. Furthermore, the modularity Mod is introduced to judge 
the quality of community detection under the different numbers of communities. The modularity of the number of communities from four to eight is shown in Table 1.

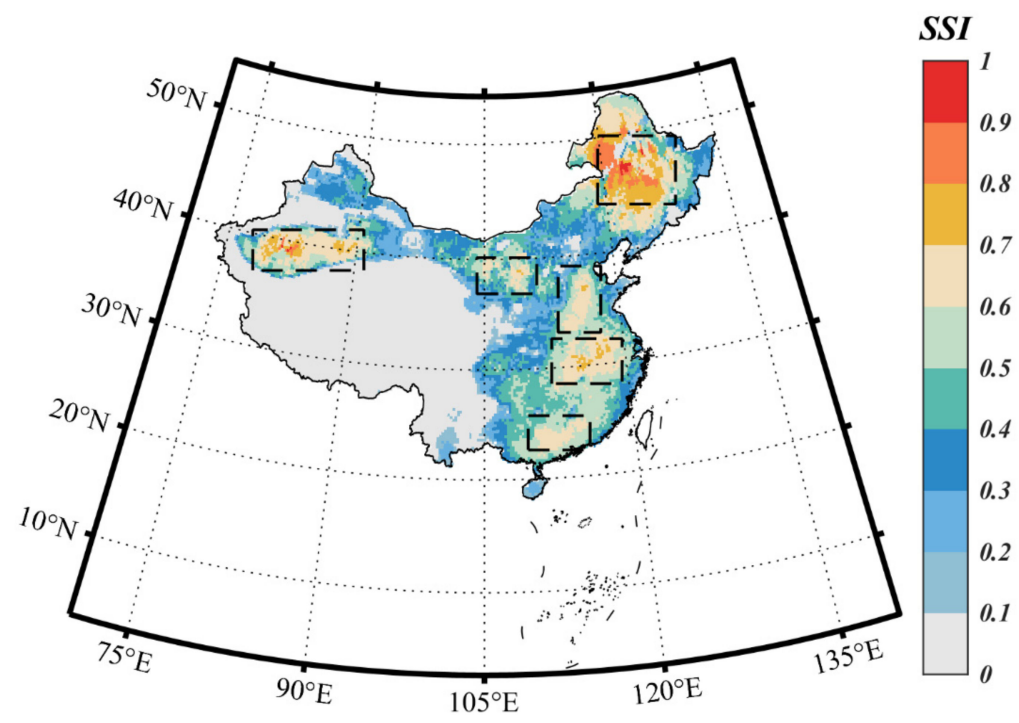

Figure 7. The synchronization strength index (SSI) of nodes. Black dashed box indicates regions where the $S S I \in[0.6,1.0]$.

Table 1. The modularity under the different numbers of communities.

\begin{tabular}{cccccc}
\hline The Number of Communities & $\mathbf{4}$ & $\mathbf{5}$ & $\mathbf{6}$ & $\mathbf{7}$ & $\mathbf{8}$ \\
\hline The modularity Mod & 0.667 & 0.680 & 0.687 & 0.691 & 0.688 \\
\hline
\end{tabular}

We found that the highest value of Mod occurs when the number of communities is seven. With this number of communities, the northern regions of NWC and southern regions of SWC are classified as same community. However, spatial verification shows that this teleconnection does not exist; thus, we chose eight, corresponding to the second highest Mod, as the number of communities for subsequent community detection. Meanwhile, in order to ensure the local optimum of community detection, we also calculated the modularity when the number of communities was 9,10 and 11 , and found that the modularity decreases as the number of communities increases. The results of community detection with eight communities are shown in Figure 8. It can be seen that community 1, 2, 3, 5, and 7 contain six core regions, indicating that the proposed SSI is indicative for the initial determination of the number of communities in the spin glass algorithm, and the spin glass algorithm further discovers the closely related components in the synchronization strength network and provides clear boundaries. Each community can provide the propagation extent of CDHEs, and the total number of CDHEs is relatively close between nodes within a community.

$\mathrm{AMO}$ and PDO have been proven to influence the formation of CDHEs in China $[23,28]$. In this study, we correlate a temporal series of the mean frequency and mean magnitude within different communities with a temporal series of AMO and PDO indexes during JJA, aiming to explore the driving factors of CDHEs in different regions of China. The results of the correlation analysis are presented in Tables 2 and 3. As shown in Tables 2 and 3, the variations in the mean frequency and mean magnitude of CDHEs within community 1 and 2 are significantly and positively correlated with the AMO index, implying that the AMO pattern may modulate variations in the frequency and magnitude of CDHEs within community 1 and 2, and that the frequency and magnitude of CDHEs within community 2 are more positively modulated by the AMO pattern. In community 7 and 8 , which are also located in the northern China, variations in the mean frequency and mean magnitude of CDHEs are not significantly correlated with individual patterns, but variations in the 
mean magnitude of CDHEs in community 8 are significantly correlated with the compounding effect of $\mathrm{AMO}$ and $\mathrm{PDO}$; the results of the standardized regression coefficients for individual patterns show that AMO plays a dominant role in positively modulating variations in the mean magnitude of CDHEs in community 8 . Similarly, variations in the mean magnitude of CDHEs in community 6, located in the southern regions of SWC, are significantly correlated with the compounding effect of AMO and PDO, and AMO also dominates in positively modulating variations in the mean magnitude of community 6 . Previous studies [23] have shown that the warm $\mathrm{AMO}$ phase tends to increase the frequency of CDHEs in northern China and the southern regions of SWC, and AMO is significantly and positively correlated with temperature in most of these regions. When $\mathrm{AMO}$ is in the warm phase, the warming of the northern China and the southern regions of SWC may lead to a high magnitude of CDHEs; this also explains the positive modulation of AMO in the frequency and magnitude of CDHEs in communities located in the northern China and the southern regions of SWC. During the warm phase of AMO, the western North Atlantic releases more heat flux from the ocean into the atmosphere, which triggers two types of stationary wave trains that propagate eastward. One part is an arching wave train that propagates from the western North Atlantic toward the polar region and even farther into northeastern Asia (the positive polar-Eurasian pattern), and the other part is a Rossby wave train zonally propagating from the western North Atlantic to East Asia (the negative Silk Road pattern). These wave trains form a downward motion in most of China, providing favorable conditions for high temperatures and precipitation deficits; when AMO is in the cold phase, the reverse applies [46]. Community 3 is located in the central-eastern region of China, where the correlations between the mean frequency of CDHEs and individual patterns are negative and none of them are significant, but the mean frequency of CDHEs is significantly and positively correlated with the compounding effect of AMO and PDO, which indicates that $\mathrm{AMO}$ and PDO jointly influence the variations in the frequency of CDHEs in this region. According to the results of the standardized regression coefficients, the contribution of AMO to the variations in the mean frequency of CDHEs is greater than that of PDO. For the variation in the magnitude of CDHEs within community 3, the AMO pattern is significantly negatively correlated with the variation in the mean magnitude of CDHEs. AMO has a significant positive correlation with precipitation and a non-significant positive correlation with temperature in the central-eastern region of China. The increase (decrease) in precipitation may inhibit (promote) the formation of CDHEs and decrease (increase) the magnitude of CDHEs. Therefore, AMO may negatively modulate variations in the frequency and magnitude of CDHEs, which is consistent with the results in this study.

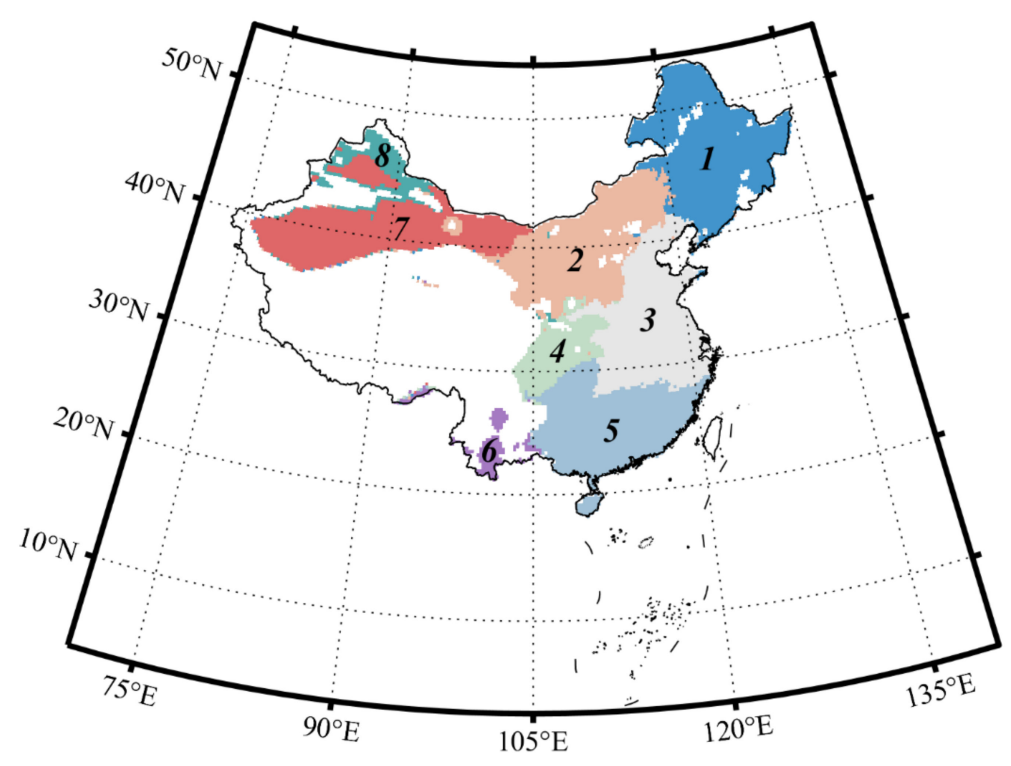

Figure 8. The spatial distribution of communities when the number of communities is 8 . 
Table 2. The single and multiple correlation coefficient between temporal series of AMO, PDO index during JJA and the mean frequency of CDHEs in 8 communities.

\begin{tabular}{|c|c|c|c|c|c|c|c|c|}
\hline & & & & & & & & \\
\hline \multicolumn{9}{|l|}{ Teleconnection Pattern } \\
\hline $\mathrm{AMO}$ & 0.27 * & $0.40^{* *}$ & -0.23 & 0.05 & -0.06 & 0.15 & 0.07 & 0.13 \\
\hline PDO & -0.03 & -0.19 & -0.16 & -0.26 * & -0.07 & 0.06 & -0.07 & -0.10 \\
\hline $\mathrm{AMO}$ and PDO & $0.27 *$ & $0.41^{* *}$ & $0.32 *$ & 0.26 * & 0.11 & 0.18 & 0.09 & 0.15 \\
\hline
\end{tabular}

Note: the significance level of correlation $p<0.01$ is denoted as ${ }^{* *}, 0.01<p<0.05$ is denoted as *

Table 3. The single and multiple correlation coefficient between temporal series of AMO, PDO index during JJA and the mean magnitude of CDHEs in 8 communities.

\begin{tabular}{|c|c|c|c|c|c|c|c|c|}
\hline \multicolumn{9}{|c|}{ Community } \\
\hline & 1 & 2 & 3 & 4 & 5 & 6 & 7 & 8 \\
\hline $\begin{array}{r}\text { leleconnection Pattern } \\
\mathrm{AMO}\end{array}$ & $0.28 *$ & $0.48^{* *}$ & $-0.27^{*}$ & 0.06 & -0.01 & 0.10 & 0.04 & 0.25 \\
\hline PDO & -0.04 & -0.20 & -0.17 & -0.27 * & -0.09 & 0.23 & 0.05 & 0.06 \\
\hline $\mathrm{AMO}$ and PDO & $0.28 *$ & $0.49 * *$ & $0.36 * *$ & $0.27 *$ & 0.09 & $0.28 *$ & 0.07 & 0.28 * \\
\hline
\end{tabular}

Note: the significance level of correlation $p<0.01$ is denoted as $* *, 0.01<p<0.05$ is denoted as *

The variations in the mean frequency and mean magnitude of CDHEs within community 4 are significantly negatively correlated with the PDO pattern, which may be attributed to the significant negative correlation between PDO and temperature in the region. The potential mechanism of this correlation can be explained by the EAP teleconnection wave train [29]. The variations in the mean frequency and mean magnitude of CDHEs within community 5 do not significantly respond to AMO or PDO patterns. A reason for this may be that temperature and precipitation within community 5 are not sensitive to changes in $\mathrm{AMO}$ and PDO patterns [23].

\section{Conclusions}

In this study, we define CDHEs using daily maximum temperature and a monthly scale SPI index, and focus on the spatial and temporal variations in the annual frequency and annual magnitude of CDHEs during 1961-2018; this analysis shows that, on a national scale, the mean frequency and mean magnitude of CDHEs, respectively, show a nonsignificant decrease and a non-significant increase. In China, CDHEs rarely occur in areas with high altitude or low temperature. The spatial distribution of the mean annual frequency and mean annual magnitude of CDHEs is more consistent in northern China, while spatial distribution is divergent elsewhere. The trends in the annual frequency and annual magnitude of CDHEs within each grid point are also discussed, and significant increases or decreases in the annual frequency and annual magnitude can be attributed to the individual or combined effect of variations in precipitation and maximum temperature.

We further obtained the propagation extent of CDHEs through the constructed synchronization strength network combined with the proposed synchronization strength index, and discussed the correlation between the mean frequency and mean magnitude of CDHEs, AMO, and PDO within each community to explore possible physical mechanisms. $\mathrm{AMO}$, through two types of teleconnection wave trains, positively modulates the mean frequency and mean magnitude of CDHEs within community 1 and 2, and negatively modulates the mean magnitude of CDHEs within community 3. PDO negatively modulates the mean frequency and mean magnitude of CDHEs within community 4. AMO and PDO jointly modulate the mean magnitude of CDHEs within community 6 and 8 . CDHEs have a serious impact on the development of society, and there is minimal research regarding the spatial propagation direction of CDHEs, these research have positive implications for forecasting and emergency management of compound disaster events. In future research, we will study the spatial propagation of CDHEs from the perspective of complex networks. 
Author Contributions: Conceptualization, all authors; methodology, K.L. (Kaiwen Li); data collection, K.L. (Kaiwen Li); first draft, K.L. (Kaiwen Li); draft review and editing, M.W., K.L. (Kai Liu); formal analysis, K.L. (Kaiwen Li); supervision and validation, K.L. (Kaiwen Li), M.W.; investigation, all authors; Funding Acquisition, M.W., K.L. (Kai Liu). All authors have read and agreed to the published version of the manuscript.

Funding: The research for this article was supported by the National Key Research and Development Plan (2017YFC1502902).

Institutional Review Board Statement: Not applicable.

Informed Consent Statement: Not applicable.

Data Availability Statement: CN05.1 dataset used in this paper is available at the China Meteorological Data Service Center website (http:/ / data.cma.cn/, accessed on 18 November 2021).

Acknowledgments: The authors appreciate Tian Xie for his contribution to the language editing of this article.

Conflicts of Interest: The authors declare no conflict of interest.

\section{References}

1. Zhou, P.; Liu, Z. Likelihood of concurrent climate extremes and variations over China. Environ. Res. Lett. 2018, 13, 094023. [CrossRef]

2. Kirono, D.G.C.; Hennessy, K.J.; Grose, M.R. Increasing risk of months with low rainfall and high temperature in southeast Australia for the past 150 years. Clim. Risk Manag. 2017, 16, 10-21. [CrossRef]

3. Seneviratne, S.; Nicholls, N.; Easterling, D.; Goodess, C.; Kanae, S.; Kossin, J.; Luo, Y.; Marengo, J.; McInnes, K.; Rahimi, M.; et al. Changes in Climate Extremes and Their Impacts on the Natural Physical Environment: An Overview of the IPCC SREX Report; Cambridge University Press: Cambridge, UK; New York, NY, USA, 2012; ISBN 9781139177245.

4. Chen, L.; Chen, X.; Cheng, L.; Zhou, P.; Liu, Z. Compound hot droughts over China: Identification, risk patterns and variations. Atmos. Res. 2019, 227, 210-219. [CrossRef]

5. Ye, L.; Shi, K.; Xin, Z.; Wang, C.; Zhang, C. Compound Droughts and Heat Waves in China. Sustainability 2019, 11, 3270. [CrossRef]

6. García-Herrera, R.; Díaz, J.; Trigo, R.M.; Luterbacher, J.; Fischer, E.M. A Review of the European Summer Heat Wave of 2003. Crit. Rev. Environ. Sci. Technol. 2010, 40, 267-306. [CrossRef]

7. Rippey, B.R. The US drought of 2012. Weather Clim. Extrem. 2015, 10, 57-64. [CrossRef]

8. McKee, T.B.; Nolan, J.; Kleist, J. The relationship of drought frequency and duration to time scales. In Proceedings of the 8th Conference on Applied Climatology, Anaheim, CA, USA, 17-22 January 1993.

9. Yuan, X.; Jian, J.; Jiang, G. Spatiotemporal Variation of Precipitation Regime in China from 1961 to 2014 from the Standardized Precipitation Index. ISPRS Int. J. Geo-Inf. 2016, 5, 194. [CrossRef]

10. Vicente-Serrano, S.M.; Beguería, S.; López-Moreno, J.I. A Multiscalar Drought Index Sensitive to Global Warming: The Standardized Precipitation Evapotranspiration Index. J. Clim. 2010, 23, 1696-1718. [CrossRef]

11. Liu, C.; Yang, C.; Yang, Q.; Wang, J. Spatiotemporal drought analysis by the standardized precipitation index (SPI) and standardized precipitation evapotranspiration index (SPEI) in Sichuan Province, China. Sci. Rep. 2021, 11, 1280. [CrossRef]

12. Wells, N.; Goddard, S.; Hayes, M.J. A self-calibrating Palmer Drought Severity Index. J. Clim. 2004, 17, 34-46. [CrossRef]

13. Zhao, H.; Gao, G.; An, W.; Zou, X.; Li, H.; Hou, M. Timescale differences between SC-PDSI and SPEI for drought monitoring in China. Phys. Chem. Earth 2017, 102, 48-58. [CrossRef]

14. Xu, Z.; Fitzgerald, G.; Guo, Y.; Jalaludin, B.; Tong, S. Impact of heatwave on mortality under different heatwave definitions: A systematic review and meta-analysis. Environ. Int. 2016, 89-90, 193-203. [CrossRef]

15. Tong, S.; Kan, H. Heatwaves: What is in a definition? Maturitas 2011, 69, 5-6. [CrossRef]

16. Hao, Z.; Hao, F.; Singh, V.P.; Zhang, X. Quantifying the relationship between compound dry and hot events and El Niño-southern Oscillation (ENSO) at the global scale. J. Hydrol. 2018, 567, 332-338. [CrossRef]

17. Olmo, M.; Bettolli, M.L.; Rusticucci, M. Atmospheric circulation influence on temperature and precipitation individual and compound daily extreme events: Spatial variability and trends over southern South America. Weather. Clim. Extremes 2020, 29, 100267. [CrossRef]

18. Geirinhas, J.L.; Russo, A.; Libonati, R.; Sousa, P.M.; Miralles, D.G.; Trigo, R.M. Recent increasing frequency of compound summer drought and heatwaves in Southeast Brazil. Environ. Res. Lett. 2021, 16, 034036. [CrossRef]

19. Wu, X.; Hao, Z.; Hao, F.; Singh, V.P.; Zhang, X. Dry-hot magnitude index: A joint indicator for compound event analysis. Environ. Res. Lett. 2019, 14, 064017. [CrossRef]

20. Wu, X.; Hao, Z.; Zhang, X.; Li, C.; Hao, F. Evaluation of severity changes of compound dry and hot events in China based on a multivariate multi-index approach. J. Hydrol. 2020, 583, 124580. [CrossRef]

21. Wu, X.; Hao, Z.; Hao, F.; Zhang, X. Variations of compound precipitation and temperature extremes in China during 1961-2014. Sci. Total. Environ. 2019, 663, 731-737. [CrossRef] 
22. Mukherjee, S.; Ashfaq, M.; Mishra, A.K. Compound Drought and Heatwaves at a Global Scale: The Role of Natural Climate Variability-Associated Synoptic Patterns and Land-Surface Energy Budget Anomalies. J. Geophys. Res. Atmos. 2020, 125, e2019JD031943. [CrossRef]

23. Wu, X.; Hao, Z.; Hao, F.; Zhang, X.; Singh, V.P.; Sun, C. Influence of Large-Scale Circulation Patterns on Compound Dry and Hot Events in China. J. Geophys. Res. Atmos. 2021, 126, 1-15. [CrossRef]

24. Boers, N.; Bookhagen, B.; Barbosa, H.D.M.J.; Marwan, N.; Kurths, J.; Marengo, J.A. Prediction of extreme floods in the eastern Central Andes based on a complex networks approach. Nat. Commun. 2014, 5, 5199. [CrossRef]

25. Boers, N.; Goswami, B.; Rheinwalt, A.; Bookhagen, B.; Hoskins, B.; Kurths, J. Complex networks reveal global pattern of extreme-rainfall teleconnections. Nature 2019, 566, 373-377. [CrossRef]

26. Konapala, G.; Mishra, A. Review of complex networks application in hydroclimatic extremes with an implementation to characterize spatio-temporal drought propagation in continental USA. J. Hydrol. 2017, 555, 600-620. [CrossRef]

27. $\mathrm{Wu}, \mathrm{J} . ; \mathrm{Gao}, \mathrm{X} . J$. A gridded daily observation dataset over China region and comparison with the other datasets. Chin. J. Geophys. 2013, 56, 1102-1111. [CrossRef]

28. Li, H.; He, S.; Gao, Y.; Chen, H.; Wang, H. North Atlantic Modulation of Interdecadal Variations in Hot Drought Events over Northeastern China. J. Clim. 2020, 33, 4315-4332. [CrossRef]

29. Zhang, G.; Zeng, G.; Li, C.; Yang, X. Impact of PDO and AMO on interdecadal variability in extreme high temperatures in North China over the most recent 40-year period. Clim. Dyn. 2020, 54, 3003-3020. [CrossRef]

30. Zuo, J.; Pullen, S.; Palmer, J.; Bennetts, H.; Chileshe, N.; Ma, T. Impacts of heat waves and corresponding measures: A review. J. Clean. Prod. 2015, 92, 1-12. [CrossRef]

31. Russo, S.; Dosio, A.; Graversen, R.G.; Sillmann, J.; Carrao, H.; Dunbar, M.B.; Singleton, A.; Montagna, P.; Barbola, P.; Vogt, J.V. Magnitude of extreme heat waves in present climate and their projection in a warming world. J. Geophys. Res. Atmos. 2014, 119, 12-500. [CrossRef]

32. Yevjevich, V.; Ica Yevjevich, V. An Objective Approach to Definitions and Investigations of Continental Hydrologic Drought $\mathrm{s}$ an Objective Approach to Definitions and Investigations of Continental Hydrologic Droughts. Ph.D. Thesis, Colorado State University, Fort Collins, CO, USA, 1967.

33. Nam, W.-H.; Hayes, M.J.; Svoboda, M.D.; Tadesse, T.; Wilhite, D.A. Drought hazard assessment in the context of climate change for South Korea. Agric. Water Manag. 2015, 160, 106-117. [CrossRef]

34. Quiroga, R.Q.; Kreuz, T.; Grassberger, P. Event synchronization: A simple and fast method to measure synchronicity and time delay patterns. Phys. Rev. E 2002, 66, 041904. [CrossRef] [PubMed]

35. Li, Y.; Shang, Y.; Yang, Y. Clustering coefficients of large networks. Inf. Sci. 2017, 382-383, 350-358. [CrossRef]

36. Masuda, N.; Sakaki, M.; Ezaki, T.; Watanabe, T. Clustering Coefficients for Correlation Networks. Front. Aging Neurosci. 2018, 12, 7. [CrossRef] [PubMed]

37. Newman, M.E.J.; Girvan, M. Finding and evaluating community structure in networks. Phys. Rev. E 2004, 69, 026113. [CrossRef]

38. Girvan, M.; Newman, M.E.J. Community structure in social and biological networks. Proc. Natl. Acad. Sci. USA 2002, 99, 7821-7826. [CrossRef]

39. Pons, P.; Latapy, M. Computing Communities in Large Networks Using Random Walks. J. Graph Algorithms Appl. 2006, 10, 191-218. [CrossRef]

40. Zhu, X.; Ghahramani, Z. Learning from labeled and unlabeled data with label propagation. Sch. Comput. Sci. Carnegie Mellon Univ. Tech. Rep. C 2002, 3175, 237-244. [CrossRef]

41. Reichardt, J.; Bornholdt, S. Statistical mechanics of community detection. Phys. Rev. E 2006, 74, 016110. [CrossRef]

42. Haq, N.F.; Moradi, M.; Wang, Z.J. Community structure detection from networks with weighted modularity. Pattern Recognit. Lett. 2019, 122, 14-22. [CrossRef]

43. Ayantobo, O.O.; Li, Y.; Song, S.; Yao, N. Spatial comparability of drought characteristics and related return periods in mainland China over 1961-2013. J. Hydrol. 2017, 550, 549-567. [CrossRef]

44. Yao, N.; Li, Y.; Lei, T.; Peng, L. Drought evolution, severity and trends in mainland China over 1961-2013. Sci. Total Environ. 2018, 616-617, 73-89. [CrossRef]

45. Cao, L.; Zhu, Y.; Tang, G.; Yuan, F.; Yan, Z. Climatic warming in China according to a homogenized data set from 2419 stations. Int. J. Clim. 2016, 36, 4384-4392. [CrossRef]

46. Zhu, Y.; Wang, T.; Ma, J. Influence of internal decadal variability on the summer rainfall in Eastern China as simulated by CCSM4. Adv. Atmospheric Sci. 2016, 33, 706-714. [CrossRef] 